\title{
Brote familiar de piodermitis por Staphylococcus aureus meticilino-resistente adquirido en la comunidad
}

\section{Family outbreak of methicillin-resistant Staphylococcus aureus pyodermitis acquired in the community}

Rodrigo Mauricio Cruz Choappa ${ }^{1 \underline{\underline{M}}}$, Miranda Andrea Ocara Vargas² ${ }^{2}$ Cesar Rodolfo Haro Schaper ${ }^{3}$

1 Profesor titular Universidad de Valparaíso y Médico Infectólogo adultos, Hospital Carlos van Buren de Valparaíso. Chile.

${ }^{2}$ Alumna de sexto año de Medicina, ayudante de infectología Universidad de Valparaíso. Chile.

${ }^{3}$ Médico Infectológo pediátrico, Hospital Carlos van Buren de Valparaíso. Chile.

Fecha correspondencia:

Recibido: febrero 04 de 2021.

Revisado: marzo 16 de 2021.

Aceptado: mayo 10 de 2021.

Forma de citar:

Cruz Choappa R, Ocara M, Haro C. Brote familiar de piodermitis por Staphylococcus aureus meticilinoresistente adquirido en la comunidad. Rev CES Med. 2021; 35(2): 126-134. 10.21615/

cesmedicina. 6085

\section{Open access}

(C) Derecho de autor

Licencia creative commons

Ética de publicaciones

Revisión por pares

Gestión por Open Journal System

DOI: http://dx.doi.org/10.21615/

cesmedicina.6085

ISSNe 2215-9177

ISSN 0120-8705

Publica con nosotros

\section{Resumen}

Las infecciones por Staphylococcus aureus meticilino-resistente adquiridas en la comunidad son cada vez más frecuentes y se deben sospechar en casos de piodermitis $u$ otras infecciones necrotizantes, tanto en niños como en adultos sin factores de riesgo clásicos. Se presenta un brote familiar de piodermitis por Staphylococcus aureus meticilino-resistente adquirido en la comunidad, ocurrido en la ciudad de Valparaíso-Chile, que afectó a una madre de veintiséis años y a su hijo de diez quienes debieron ser tratados con aseo quirúrgico y cotrimoxazol forte, con buena respuesta clínica.

Palabras clave: Piodermitis; Staphylococcus aureus; Comunidad; Brote.

\section{Abstract}

Community-acquired methicillin-resistant Staphylococcus aureus infections are becoming more frequent and should be suspected in cases of pyoderma or other necrotizing infections, both in children and adults without classic risk factors. A family outbreak of pyodermitis by Community-acquired methicillin-resistant 
Staphylococcus aureus infection occurred in Valparaíso-Chile, which affected a twenty-six-yearold mother and her ten-year-old son, who had to be treated with a surgical toilet and cotrimoxazole forte, with good clinical response.

Palabras clave: Pyoderma; Staphylococcus aureus; Community; Outbreak.

\section{Introducción}

Las infecciones cutáneas pueden comenzar como foliculitis, forunculosis o celulitis, pudiendo progresar hacia el tejido subcutáneo más profundo en cualquier región del cuerpo ${ }^{(1,2)}$. Varios organismos que normalmente colonizan la piel pueden causar necrosis y licuefacción, con la acumulación posterior de leucocitos y desechos celulares, conduciendo a la formación de abscesos $^{(1,2)}$. Staphylococcus aureus y Streptococcus pyogenes son los agentes más frecuentemente aislados en las distintas infecciones de piel y partes blandas ${ }^{(2,3)}$. Otros agentes importantes, aunque menos frecuentes son Streptococcus del grupo B, C y G, Enterococcus spp., bacilos gram negativos y anaerobios ${ }^{(3)}$.

Varios estudios han evidenciado el aumento de cepas de $S$. aureus meticilino resistente (SAMR) como causante de infecciones purulentas de piel y partes blandas ${ }^{(3-5)}$. SAMR se describió por primera vez en Inglaterra en 1961 poco después de la introducción de la meticilina ${ }^{(6)}$. Desde entonces, SAMR se ha extendido por todo el mundo y su prevalencia ha aumentado tanto en las infecciones intrahospitalarias como comunitarias ${ }^{(7,8)}$.

La resistencia a la meticilina está mediada por la proteína de unión a penicilina 2a, codificada en el gen mecA que permite que este microrganismo crezca y se reproduzca en presencia de meticilina y otros antibióticos beta-lactámicos. El gen mecA se encuentra en un elemento genético móvil llamado cassette del cromosoma estafilocócico (SCCmec) $)^{(4,5)}$.

En la década de 1990 se describió a S. aureus meticilino resistente adquirido en la comunidad (SAMR-AC) como patógeno en individuos sin factores de riesgo (hemodiálisis, cirugía, hospitalización reciente, portadores de dispositivos percutáneos, infección previa por SAMR, estadía en hogar o asilo de ancianos en el último año). SAMR-AC posee características distintas a las cepas nosocomiales, ya que presenta resistencia a menos antimicrobianos, no se asocia a los factores de riesgo clásicos, posee una base genética y factores de virulencia distintos, producción de leucocidina de panton valentine (LPV), además de provocar, principalmente, infecciones abscedadas de piel y neumonía necrotizante ${ }^{(8,9)}$. 
Se han descrito brotes comunitarios por este agente principalmente en grupos de niños, hombres que tenían sexo con hombres, equipos de jugadores de deportes de contacto e internos de cárceles ${ }^{(10,11)}$. En Chile, se ha reportado el aislamiento de SAMR-AC tanto en niños como en adultos desde 2006, provocando infecciones superficiales y profundas ${ }^{(12,13)}$.

El objetivo de este trabajo es presentar un brote familiar de piodermitis por SAMR-AC, revisar la epidemiología, presentación clínica, diagnóstico y tratamiento.

\section{Descripción del brote}

El 10 de octubre de 2018 consultó en la unidad de emergencia de adultos del Hospital Carlos van Buren de la quinta región de Valparaíso una mujer de 26 años, sin antecedentes mórbidos, quien trabajaba manipulando pescados en una céntrica calle de la ciudad y que vivía en condiciones de hacinamiento con su familia y la de su hermano (cuatro adultos y dos niños). Presentaba lesiones pustulosas y abscesos de piel en el tronco y extremidades de dos semanas de evolución (figuras 1 y 2), sin compromiso de mucosas, sin fiebre, con PCR de $125 \mathrm{mg} / \mathrm{L}$, recuento de leucocitos de 14300 células, además de anemia hipocrómica leve.

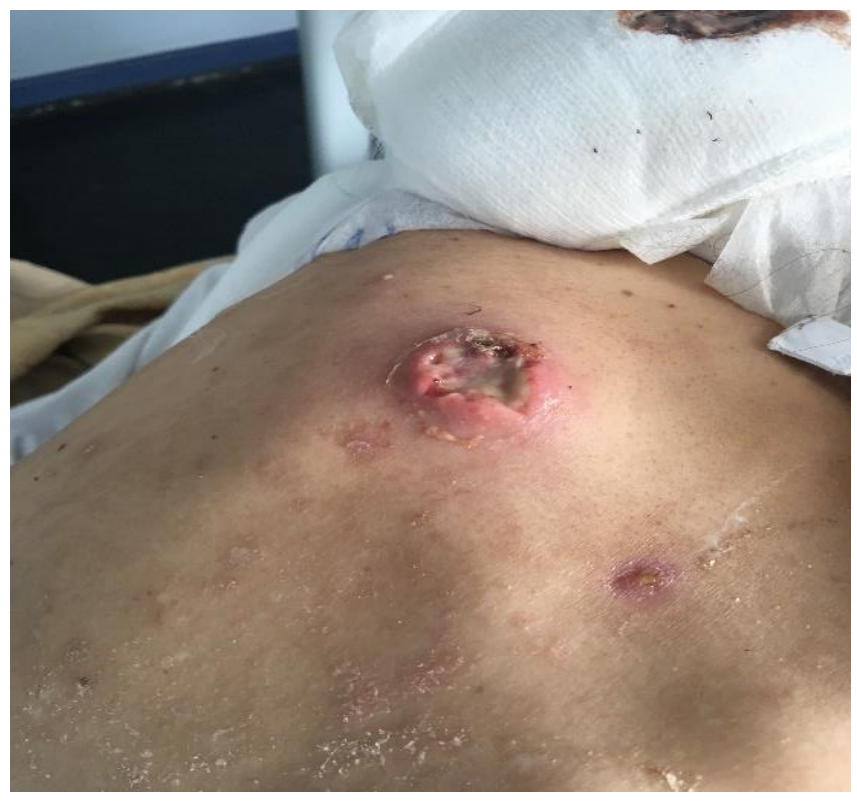

Figura 1. Abscesos en región escapular izquierda 


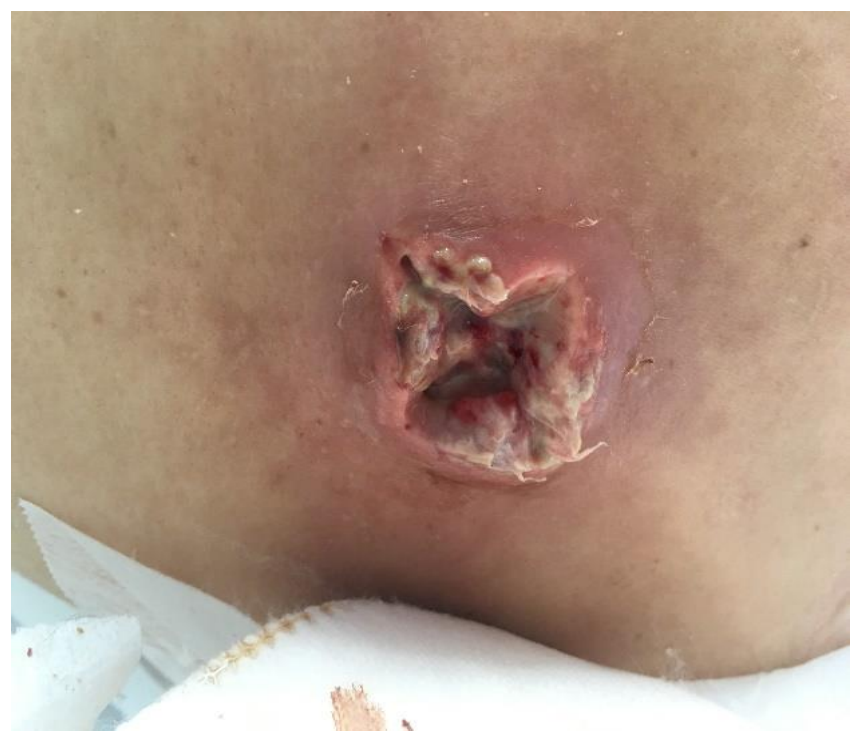

Figura 2. Absceso en región lumbar

Se realizó aseo quirúrgico y toma de cultivo de secreción y de tejido, además de iniciar tratamiento con ampicilina-sulbactam 1,5 g cada ocho horas. Por mala respuesta clínica se revisaron los cultivos, los cuales informaron desarrollo de SAMR, sensible a vancomicina, clindamicina, cotrimoxazol, ciprofloxacino, rifampicina y tetraciclina. En los hemocultivos no hubo desarrollo bacteriano. Debido a esto se cambió tratamiento a cotrimoxazol forte cada ocho horas con buena respuesta clínica (figuras 3 y 4 ).



Figura 3. Lesión escapular izquierda luego de 10 días de tratamiento con cotrimoxazol forte 


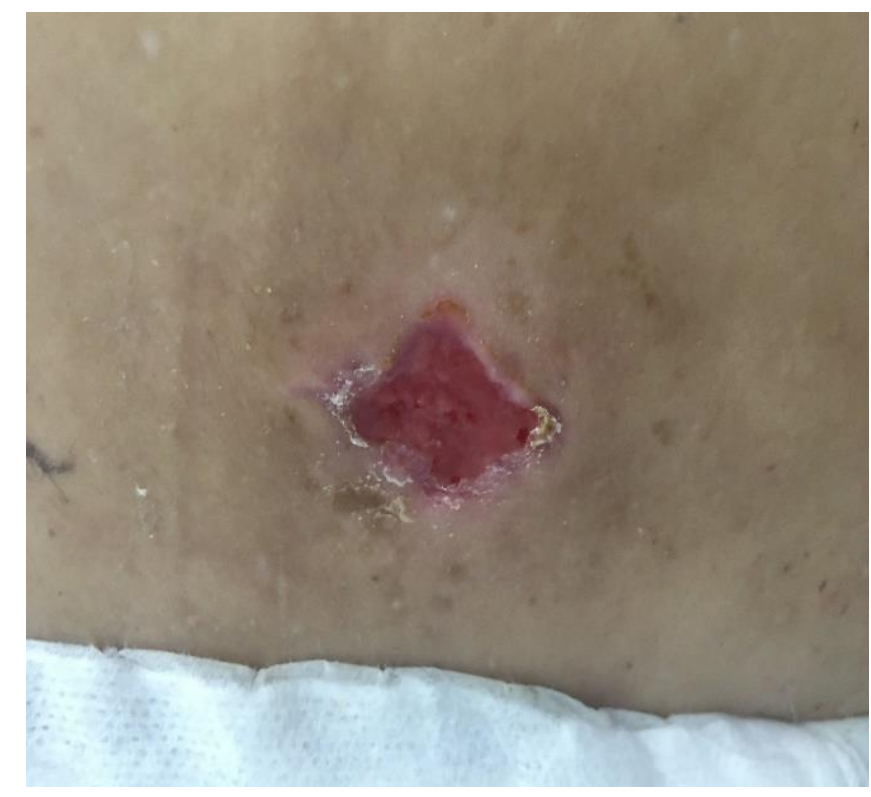

Figura 4. Lesiones en región lumbar luego de 10 días de tratamiento con cotrimoxazol forte

Se realizó ecocardiograma, el cual no mostró vegetaciones endocárdicas. La paciente fue dada de alta en buenas condiciones generales, con indicación de completar tratamiento por 14 días en su domicilio. La cepa aislada fue enviada al Instituto de Salud Pública de Chile (ISP), informándose SAMR portadora del gen de LPV.

A la interrogación dirigida y revisión de antecedentes, su hijo de 10 años, asmático y alérgico al chocolate, estuvo hospitalizado dos meses antes en el mismo centro por un cuadro que comenzó el 31 de julio con una pápula axilar derecha que evolucionó con aumento de tamaño progresivo y dolor. Consultó el 02/08 en la unidad de emergencia infantil donde se diagnosticó una celulitis y comenzó tratamiento con flucloxacilina $500 \mathrm{mg}$ cada seis horas. Alcanzó a recibir cuatro dosis antes de volver a consultar y ser hospitalizado por aumento de la signología local, fiebre $\left(38^{\circ} \mathrm{C}\right)$, con leucocitosis de 17400 células y $68 \%$ neutrófilos y PCR de 34,8 mg/l. Recibió cloxacilina $100 \mathrm{mg} / \mathrm{kg} /$ día, respondiendo parcialmente, hasta que el 07 de agosto se drenó un absceso axilar y se cultivó su contenido. Ese día se suspendió la cloxacilina y se fue de alta el 09/08, completando tratamiento oral con cefadroxilo (500 mg cada 12 horas) por ocho días. 
Evolucionó con resolución ad integrum, y con posterioridad, en control ambulatorio, se rescató el resultado del cultivo, el cual fue informado como SAMR con patrón de susceptibilidad idéntico al de su madre, por lo que recibió 14 días de tratamiento con cotrimoxazol forte cada 12 horas. No se pudo realizar estudio de clonalidad debido a que no se contó con la cepa de la infección del hijo de la paciente.

Además, relató que su cuñada también había presentado lesiones similares a las de ella los primeros días de septiembre del mismo año, las cuales fueron tratadas de forma ambulatoria en la atención primaria con varios esquemas antimicrobianos, entre ellos cotrimoxazol forte.

\section{Discusión}

Las infecciones por SAMR son cada vez más frecuentes en los distintos países, tanto a nivel hospitalario como en infecciones comunitarias ${ }^{(5-7)}$. En 2006 se publicaron en Chile los primeros cinco casos de infección de piel por SAMR-AC en adultos, quienes en su mayoría tenían el antecedente de haber viajado al extranjero ${ }^{(12)}$ y en 2015, cuatro casos pediátricos de osteomielitis, celulitis y un absceso perirrenal ${ }^{(13)}$.

Desde entonces, en nuestro país las infecciones por este agente han afectado tanto a adultos como a niños, con una incidencia que aún es baja pero que va en aumento ${ }^{(13)}$. Según reporta el ISP, en el período 2012 - 2016, se estudiaron 1079 cepas de $S$. aureus, de las cuales 90,2 \% fueron confirmadas como SAMR y el 3,7 \% (36/973) fueron derivadas desde la región de Valparaíso ${ }^{(14)}$. El 29,4 \% de las cepas de SAMR estudiadas resultaron con presencia del gen LPV, confirmándose como SAMR-AC ${ }^{(14)}$. En el brote descrito, las cepas aisladas, tanto por la ausencia de factores de riesgo, como por el patrón de susceptibilidad y la presencia del gen LPV, sugirieron que eran SAMR-AC.

Según el mismo reporte del ISP, el 50,3 \% (144/286) de las cepas de SAMR-AC PVL positivos fueron aisladas a partir de muestras de heridas, el $30,8 \%$ de abscesos y el $12,2 \%$ de hisopado nasofaríngeo ${ }^{(14)}$. Esto coincide con los casos aquí descritos, ya que las infecciones supuradas de piel son las más frecuentes, no sólo en Chile, sino en todos los países ${ }^{(15)}$.

De las cepas SAMR-AC PVL positivos y reportadas por el ISP, ninguna cepa presentó resistencia a clindamicina o cotrimoxazol y el $17 \%$ fue resistente a ciprofloxacino ${ }^{(14)}$; esto coincide con las cepas descritas aquí, las cuales eran susceptibles a estos tres antibacterianos. 
La elección del tratamiento antibiótico debe ser realizada sobre la base de los datos microbiológicos disponibles a nivel local y considerando fundamentalmente los sitios de infección, así como la gravedad del paciente. El tratamiento de elección para infecciones supuradas de piel y partes blandas es cotrimoxazol, pero también pueden ser útiles vancomicina, linezolid, daptomicina, clindamicina, ceftarolina y tigeciclina. En infecciones graves, en especial si la cepa es productora de LPV, se sugiere combinaciones de antibióticos, como por ejemplo vancomicina, linezolid, clindamicina o rifampicina ${ }^{(16)}$.

Los pacientes fueron tratados con cotrimoxazol por vía oral con buena respuesta clínica, debido a que no presentaron infecciones profundas ni sepsis grave, aunque para el niño, el drenaje del absceso fue curativo.

En conclusión, consideramos importante sospechar la presencia de SAMR-AC en infecciones comunitarias, supuradas de piel y partes blandas en pacientes sin factores de riesgo clásico, realizar aseo quirúrgico con toma de muestra para identificación y estudio de susceptibilidad para los antimicrobianos frecuentemente utilizados.

Limitaciones del estudio: es un brote comunitario de solo tres casos, donde sólo en dos de ellos se obtuvo cultivos y no se pudo realizar estudio de clonalidad por contar solo con el cultivo de la paciente del caso clínico.

\section{Aspectos éticos}

Se contó con la autorización escrita de la paciente para publicar sus antecedentes e imágenes de forma anónima y respetando la ley de deberes y derechos de los pacientes de Chile.

\section{Conflicto de Interés}

Los autores declaran no tener conflictos de interés.

\section{Fuentes de financiamiento}

No se requirió financiamientos.

\section{Bibliografía}

1. Guet-Revillet H, Jais J-P, Ungeheuer M-N, Coignard-Biehler H, Duchatelet S, Delage $M$, et al. The microbiological landscape of anaerobic infections in Hidradenitis Suppurativa: A 
Prospective Metagenomic Study. CID. 2017; 65 (2), 15: 282-91, https://doi.org/10.1093/cid/cix285.

2. Brook I. The role of anaerobic bacteria in cutaneous and soft tissue abscesses and infected cysts. Anaerobe. 2007; 13: 171-7. DOI: 10.1016/j.anaerobe.2007.08.004.

3. Valderrama-Beltrán S, Cortés J, Caro M, Cely-Andrade L, Osorio-Pinzón J, Gualtero S, et al. Guía de práctica clínica para el diagnóstico y manejo de las infecciones de piel y tejidos blandos en Colombia. Infectio. 2019; 23(4): 318-46. DOI: http://dx.doi.org/10.22354/in.v23i4.805.

4. Kong EF, Johnson JK, Jabra-Rizk MA. Community-Associated Methicillin-Resistant Staphylococcus aureus: An Enemy amidst Us. PLoS Pathog.2016;12(10):e1005837. https://doi.org/10.1371/journal.ppat.1005837.

5. Aguayo-Reyes A, Quezada-Aguiluz M, Mella S, Riedel G, Opazo-Capurro A, Bello-Toledo $\mathrm{H}$, et al. Bases moleculares de la resistencia a meticilina en Staphylococcus aureus. Rev Chil Infectol. 2018; 35 (1): 7-14. http://dx.doi.org/10.4067/s0716-10182018000100007.

6. Barber M. Methicillin-resistant staphylococci. J Cin Pathol. 1961;14: 385-93. DOI: 10.1136/jcp.14.4.385.

7. Loewen K, Schreiber Y, Kirlew M, Bocking N, Kelly L. Community-associated methicillinresistant Staphylococcus aureus infection. CFM.2017; 63 (7): 512-20.

8. Guillén R, Carpinelli L, Rodríguez F, Castro H, Quínónez B, Campuzano A, et al. Staphylococcus aureus adquiridos en la comunidad: caracterización clínica, fenotípica y genotípica de aislados en niños paraguayos. Rev Chil Infectol. 2016;33 (6): 609-18. http://dx.doi.org/10.4067/S0716-10182016000600002.

9. Otter JA, French GL. 2011. Community-associated meticillin-resistant Staphylococcus aureus strains as a cause of healthcare-associated infection. J Hosp Infect 79:189-93. DOI: 10.1016/j.jhin.2011.04.028.

10. Centers for Disease Control and Prevention. Public health dispatch: outbreaks of community-associated methicillinresistant Staphylococcus aureus skin infections -Los Angeles County, California, 2002-2003. JAMA. 2003; 289:1377. doi: 10.1001/jama.289.11.1377.

11. Paganini H, Della Latta MP, Muller Opet B, Ezcurra G, Uranga M, Aguirre C, et al. Community-acquired methicillin-resistant Staphylococcus aureus infections in children: 
multicenter trial. Arch Argent Pediatr. 2008;106: 397-403. DOI: 10.1590/S032500752008000500005.

12. Noriega LM, González P, Hormazábal JC, Pinto C, Canals M, Munita JM, et al. Community acquired infections with methicillin resistant strains of Staphylococcus aureus: report of five cases. Rev Med Chil. 2008;136(7):885-91. http://dx.doi.org/10.4067/S003498872008000700010 .

13. Acuña $M$, Benadof $D$, Jadue C, Hormazábal JC, Alarcón $P$, Contreras J, et al. Staphylococcus aureus resistente a meticilina asociado a la comunidad (SARM-AC): comunicación de los primeros cuatro casos pediátricos descritos en Hospital de Niños Roberto del Río. Rev Chil Infectol. 2015; 32(3): 350-6. http://dx.doi.org/10.4067/S071610182015000400016.

14. ISP. Vigilancia de Staphylococcus aureus meticilina resistente adquirido en la comunidad. Chile, 2012-2016. 2017; 7 (10). https://www.ispch.cl/sites/default/files/BoletinStahylococcusResistente-20062018A $\% 20(1)$.pdf.

15. Lakhundi S, Zhang K. Methicillin-Resistant Staphylococcus aureus: Molecular characterization, evolution, and epidemiology. 2018.Clin Microbiol Rev, 31(4). e0002018. doi: 10.1128/CMR.00020-18

16. Vedia L, Lopez MJ, Scapellato P, Lopardo G, Clara L, Lista N. Tratamiento de las infecciones invasivas por Staphylococcus aureus adquirido en la comunidad. Actualizaciones en SIDA e infectología. 2014; 22 (85):53-63. https://infectologia.info/wpcontent/uploads/2016/07/ASEI-85-53-63.pdf. 